\title{
Exploring Cardiac Disease Based on ML in IOT: A Comprehensive Study
}

\author{
M.Safa ${ }^{a, 1}$, Dr. A. Pandian ${ }^{a}$ \\ ${ }^{a}$ School of computing, College of Engineering and Technology \\ SRM Institute of Science and Technology, Kattankulathur, Chennai \\ Tamil Nadu, India
}

\begin{abstract}
Every human being in today's age has high importance in a stable human life. With daily new technology is launched in a world loaded with innovative business sectors to tackle more effectively the prevalent challenges of the world. We ought to figure out if as many people as possible can be spared. The death rate of multiple non-communicable heart disease forms is rising steadily each year. Many non-communicable disorders are long-term, gradual and too serious that a patient's situation is too critical to control. This leads to a sudden heart attack for most people or learning about their condition before it is too late. In this document we suggest a cardiovascular state prediction approach for IoT and Machine Learning, which will use IoT system (sensors) to capture the data needed from the human body and move it to the cloud where data is saved with user verification. The obtained information from the human body is then normalized to quantify and forecast the total condition before AI calculations are applied to them.
\end{abstract}

\section{Introduction}

In the course of recent years, the innovation business has blast higher than ever and has Different application spaces worked with (for example clinical diagnostics [1], [2] , risk factors selection [3], [4], robotics [5], [6] and etc.). This has been even more contributed with the emergence of the internet of things [7]. In particular, an intelligent health tracking device developed primarily to check heart rate at regular intervals, taking correct steps depending on the findings. This method supersedes the conventional approach to diagnostics, in which the doctor is bounded to the patient and the appearance of the specialist is the best model for patients' prosperity. Moreover, the best benefit of this scheme is that people are not actually limited to hospitals and physically present. They are tested $24 / 7$ by smartphones and other linked devices (sensor). Furthermore, people are spared from the bother of daily checks on their doctors, giving financial benefits. When you are alone and unable to do something yourself, the most commonly encountered condition in case of heart attacks is.

\footnotetext{
${ }^{1}$ M.Safa, School of computing, College of Engineering and Technology, SRM Institute of Science and Technology, Kattankulathur, Chennai, India

Email: safam@srmist.edu.in.
} 
The evolved method therefore has the capacity to conduct proper operations, such as calling an ambulance, calling the doctor/family, etc., ensuring that the system can save vital human lives and prolong lifespan times. In addition, patients are better conscious of their illnesses across these programmes and may take an active role in managing the illness to prevent it sooner. According to the WHO, health benefits are the fundamental rights of any citizen and essentially their obligation to reach them, and the IoT position is one of the most significant developments in the field of healthcare.

\section{Internet of Everythings}

The Network or IoT can be the structure of integrated computing devices, automatic and electrical processes, objects, persons or entities with a similar identifier (UIDs) and the capacity to share statistics through organizations without the need to communicate with people or individuals. It can also be identified as an online object that can be a person with an embedment with a natural display, a grower with a biochip, carriers that have built-in sensors to caution the carrier when the tyre mass is moo, and any additional function or natural question that can be selected as an IP address that can share information over a network. Administrations in a variety of businesses continually use IoT to operate more productively, changing how consumers can maximize their consumer benefit, advance decision making, and raise market esteem. Innovative aspects of the internet of things include remote innovation, microelectromechanical (MEM) structures [9], micro-services and the internet. The mixture broke the silos between working invention and invention of information to allow for the analyses of amorphous computer-generated data for little information to improve purpose. Although Ashton's was the main sign of the IoT, the concept of the associated appliances has already been under the network and the inevitable computing of this specific name since the 1970's [7]. In an IoT setting, web-enabled main devices are given, which use embedded processors, sensors and correspondence devices to relay and operate entirely on data from their circumstances. Sensor data is collected by IoT systems through communication with an IoT gateway or other edge gadgets, and afterward shipped off the cloud to be prepared or examined locally. These gadgets interact with one another from time to time. The below is a short description of the following types of machine learning problems: 1. Classification 2. Regression 3. Clustering

\section{Classification Algorithm C4.5}

For classification, the $\mathrm{C} 4.5$ calculation is utilized. Choice trees are constructed from the same amount of training data on each tree node using $\mathrm{C} 4.5$ using the knowledge entropy principle as ID3. Then C4.5 chooses one attribute of the data what separates its example set all the more adequately into subsets advanced in one gathering or the other. The parameters are the standard knowledge benefit (entropy difference) that results in selecting an attribute for data division. There are some simple cases in this algorithm.

1. Any example in the rundown is in a similar class. It simply makes an edge hub to the choice tree to choose the class. 
2. No intelligence gain from any of the characteristics. In this case, C4.5, using the predicted class value, produces a choice hub higher up in the tree.

3. Occurrence of beforehand invisible class. Again, with the anticipated benefit, C4.5 generates a choice hub in the trees

\section{Data Mining in Neural Networks}

A parallelly distributed information processing structure composed of several processing components, i.e., a neural network. They are linked by a one-way signal channel called connections. A single output link is linked to several connections in each processing element. They are regulated learning and unaided learning. This is an essential model where an organization decides and afterward contrasts a response to each information and the objective worth. If the measured result varies from the target value, the network weights are modified in compliance with a learning guideline. e.g.: perceptron of one layer, perceptron multilayer. The Unaided learning systems can benefit from particular characteristics of problems, such as self-organizing maps. The neural network involves nonlinearity, learning capacities, input-yield planning, versatility, verification reaction, disappointment resistance, neurologic correlation, etc

\section{Naïve Bayesian Classifier}

The mathematical classificatory Naïve Baye does not presume reliance on attributes to increase the later likelihood in the class determination. Theoretically, although it will not always be the case, this classifier has a minimal error rate. Inaccuracies are due to expectations of class dependency and the lack of probability evidence available. On the basis of the formula listed above and based on such conditional probability data Bayesian classifier computes the contingent likelihood of a case having a place with each class and orders the occurrence as the class of the greatest restrictive likelihood.

\section{Induction from the Decision Tree}

The development of the choice tree is completed utilizing the C4.5 inductive calculation. This delivers a choice tree dependent on preparing information which limits the normal estimation of the quantity of information arrangement tests. Any internal tree node is a main component, while a single outgoing branch is a potential scope of that segment. The leaf hubs are the class that is allotted to an examination. The center factor in the $\mathrm{C} 4.5$ calculation is its ability to pick the necessary usefulness for every hub naturally. The function of each node is chosen to efficiently separate input samples. The measure of success is the knowledge received. By using a cutting tool, the scale and complexity of trees can be minimized. Pre-tapping and post-taking are the two techniques that are frequently used to carve. 


\section{Related Work}

Neha Anilrao Sarnaik et al. have suggested a method for implanting heart-beat monitor and a system for monitoring heart attacks in which a sensor is attached to a heart rate reader that sends it through the Internet. The device uses two loops one at the end of the patient, the other at the end of the practitioner. A sensor device is used to read and view the data on the LCD screen at heart rate and microcontroller operation [11]. Ingole Abhilasha and at. Proposed the use of Raspberry Pi to track human body temperature and pulse and give the parameters on the B+ Raspberry Pi model to a variety of users. This machine works at $-55 \mathrm{~cm}$ to $+125 \mathrm{~cm}$ and the accuracy of DS18B20 is around $0.5 \mathrm{~cm}$ [12] In this device, Pic16F77A gathers data that include cardiac beat, temperature and saline levels by means of a sensor using IoT that tracks patients everywhere. Vishal Jagtap et al. This machine uses the Wireless Internet ESP8266 and Gecko IoT modules. A device Healthcare Package using IoT has been launched by Deepika Agarwal et al. This device uses the second-generation development kit of INTEL GALILEO board

\section{Methodology}

\section{Parameters}

The heart conditions of the patients are our biggest concern, because we need to gather data from patients with cardiac disease. In fact, there are 75 parameter numbers from the previous research on cardiac disease. The findings of 14 limitations have proved to be reliable enough to continue the study on 14 only limitations. The researchers have experimented on 14 limitations in Cleveland. In comparison, we have seen a similar outcome for 12 parameters. Thus, the above 12 criteria will be adequate to estimate the patient's heart condition correctly. The following parameters are related to the sensors available.

\section{Parameters Through Manual Input}

1) Age: This paper indicates that one out of two people with age 40 are at risk of heart disease, and one in three for women. If we take the age of 70 years into account, the numbers show that for men it is one out of three and one out of four for women [7].

2) Gender: Middle-aged people suffer heart disease more often, especially two to five times higher in men compared to women.

3) Type of chest pain: the most frequent signs of various coronary diseases are chest pain. This is why one of the restrictions is selected.

4) Sleeping pulse: From the paper of J.Gomez[8] and C. Li et al. [9], we get the numbers from which it gives the idea that the vast majority of the patients biting the dust from cardiovascular breakdown have been experiencing Overweight 2.6M - 4.4M, Blood Pressure 7.1M. Which drives us to pick RBP (Resting Blood Pressure) as one of the boundaries.

5) Fat: Raised One of the is cholesterol significant reason for death among individuals saying he has a heart disease by J. Gomez et al. in [6]. 
6) Fasting blood pressure: J.Gomez [5] and C. Blood pressure. Li et al. [10] indicates the number of individuals who have experienced Overweight of $2.6 \mathrm{M}-4.4 \mathrm{M}$, Blood pressure 7.1M for most of the deaths of heart disease, which is why RBP is selected as one of the criteria. Which is why RBP is the most significant consideration.

7) Cholesterol: higher cholesterol, said in J, is one of their key causes of mortality in people with heart disease. Gómez et al. in [4].

8) Sugar levels in the blood acceleration: Speeding leads to weight loss and decreasing blood sugar levels, although it is considered normal for less than $100 \mathrm{mg} / \mathrm{dL}$. For this cause, heart prediction has been chosen as a parameter

9) Blood sugar speeding: speeding results in loss of weight or reduced blood sugar levels but is considered normal at less than $100 \mathrm{mg} / \mathrm{dL}$. The parameter was then chosen to make a guess for the patient's heart.

10) ECG rest: Electrocardiograms are ECG's norm. ECG is used for heart rhythm [8] detection. What is significant, since the cardiac condition of a patient must be respected and multiple abnormalities observed. That's ECG's supporting us. In addition, the BPM listening with the ECG can be measured.

11) Optimum heart rate: the most extreme pulse is around equivalent to the heart cadence so allows us to notice the heart work during the day.

12) Exercise involved angina: Angina is a type of chest pain torment that actuates because of the absence of blood supply to the heart. It is a side effect of coronary illness.

13) a previous high: exercise-related sT despair measured across the vertical gap of the iso-electric line to the footprint of the patient.

14) Slope: slope of the ST section of peak practice which is a coronary condition predictive value.

15) CA: a collection of large fluoroscope-coloured vessels that are a prescient worth in a 0 to 3 territory.

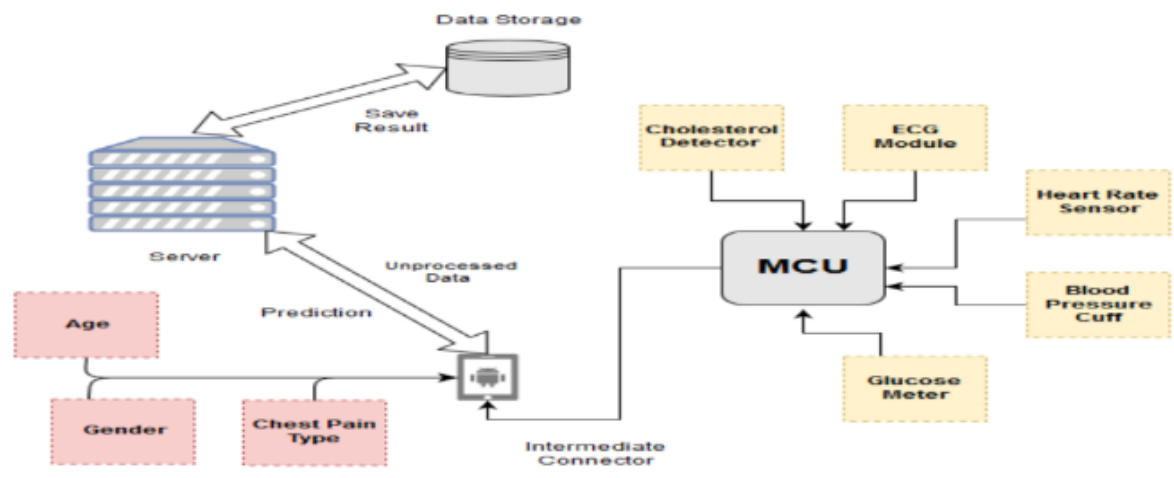

Figure 1. Combined prototype block diagram. 


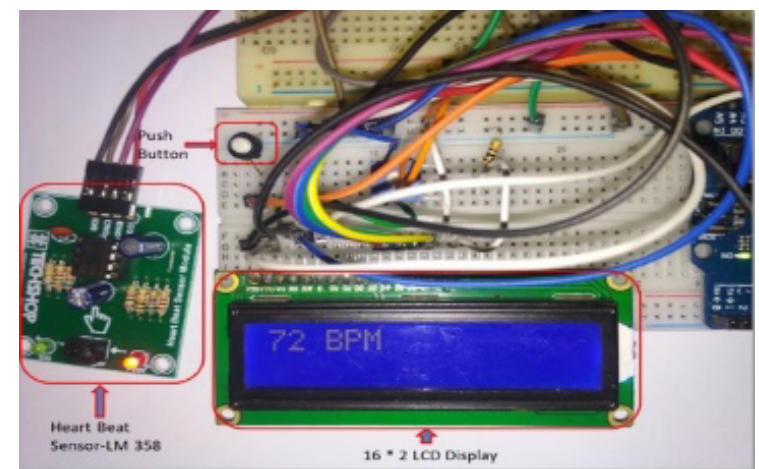

Figure 2. Implementation of a monitor for the heart rate module (LM358).

Affording to the report, those 15 limits are sufficient to quantify and forecast a patient's total heart condition, but we are focusing on the first 8 parameters because of certain This is a worked-on block graph (Figure 1) of the model wanted to be carried out. We suggested using a Microcontroller Module (MCU). The MCU is a microcontroller device is attached to sensors and components in electronics necessary. Using Bluetooth to sending the data to the smartphone after all of the hardware components have been obtained. The mobile also gathers the remaining 3 parameters. Then all 8 boundaries are shared and guided into the cloud running a calculation for ace discovering that gives the client the outcome. This is the real acknowledgment of MCU-associated Sensor Module for Heart Rate (Figure 2). The module tests the information for 10 seconds and delivers the Arduino's simple yield, which is then increased by 6 to acquire the patient's BPM. Constraints and left the exercise for future work, which involves angina, old max, pitch and CA.

The patient's cardiac disease can be demonstrated by repeated variations in heart rhythm or pulse regularity. The measured Beats per minute (BPM) for a patient in our applied module dictates whether the patient's body state is ordinary or whether the respiratory rate is extremely rapid. See the table for more detail for more information on our module. A single ECG module is staffed to calculate the electrocardiogram using 3 electrodes. First, the production pin was attached to an oscilloscope in which we had a signal detected. By analyzing a paper [1], we have correlated our signal received with the actual ECG signal. Then we also visualized the output of the serial plotter with a very promising output waveform. The most confounded piece of an ECG signal is the QRS (top worth), the Q section and the S fragment discovered in the output of our signal Nonetheless, we are attempting to develop and achieve a even better outcome with the ECG signal output. the sensor for Infrared (near-infrared) light is delivered to the human body in order to measure cholesterol without the need for blood sampling [11]. 30 seconds are taken each second, with 30 samples taken in each second. We follow up the detection protocol. We then take the average voltage decrease around the output terminal. We take cholesterol in a balanced person as a normal value of $5.2 \mathrm{~mm} / \mathrm{L}$.

\section{Proposed Algorthim}

\section{1: Start}

2: assign id to the sensor 
3: Check if Sensor are connected

4: If connected then

5: $\quad$ Proceed to step 7

6: else

7: $\quad$ Establish connection between Sensor and Node Processor

8: for Every Sensor Ss, do

9: $\quad$ for Every Parameter value do

10: Read all the value from step 5.

11: Publish value check the timing interval

12: analyses the value

13: go to the step 3

\section{Results and Discussion}

The following are the categories of benefits of IoT: higher reaction times and the capacity to give timely information for decision-making by automation. Improved planning as a result of new insights gained from larger data sets Lower operating expenses. These findings suggest to researchers and practitioners that expected benefits are frequently related to the use of IoT data, such as operational reactions to data generated by IoT sensors, and that IoT, while obviously useful for action-reaction use cases, can provide organizations with far more benefits than purely operational benefits. The values from the nodes are collected and for analysis used various analytics models and addition to that add some intelligence for improved results

\section{Graph of Temp(Celcius) Vs Time}

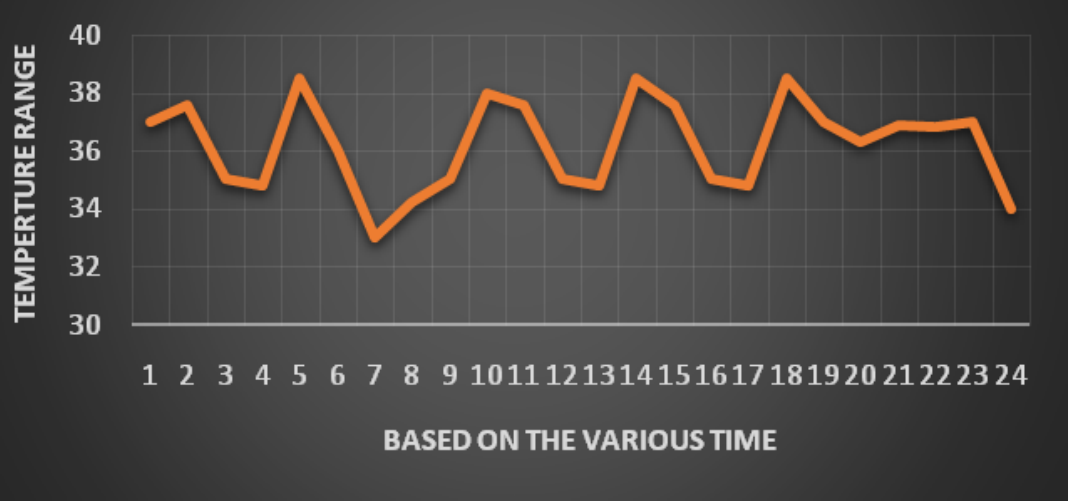

Figure 3. Graph Analysis from Temperature sensor

\section{Conclusions}

The advances in mobile and remote welfare programmes lead from the determination to the care of various disorders such as cardiovascular diseases in many areas of our lives. Android apps are now a part of the numerous options which are announced as side-byside treatment initiatives as a variety of wellness monitoring applications. We encountered some challenges during the implementation process. Due to the lack of 
supplies in our region, it was difficult to locate the actual IoT devices to enforce our requisite criteria. The feedback was tough to obtain from a photodiode during the implementation of the near-infrared sensor for cholesterol from the human body. After a lot of work, we get a photodiode electrical current and transform it into voltage. On some subjects, we tried our system and compared the true cholesterol values to the end results of a patient's cholesterol Collecting an electrocardiogram (ECG) signal from the human body introduced one more test. From the outset, the outcomes we acquired didn't appear to be in accordance with our prerequisites. Like a measure, the ECG output signal was distorted. substantially different from the previous one.

\section{Acknowledgement}

The Part time Ph. D - IT/IT Enabled Services (IT/ITES) is supported by Ministry of Electronics and Information Technology, Government of India initiated "Visvesvaraya $\mathrm{PhD}$ Scheme for Electronics and IT

\section{Reference}

[1]. F. Akhtar, J. Li, M. Azeem, S. Chen, H. Pan, Q. Wang, and J.-J. Yang, "Effective large for gestational age prediction using machine learning techniques with monitoring biochemical indicators," The Journal of Supercomputing, pp. 1-19, 2019.

[2]. F. Akhtar, Y. Pei, M. Azeem, et al., "A semi-supervised technique for lga prognosis," in Proceedings of The International Workshop on Future Technology, pp. 36-37, 2019.

[3]. F. Akhtar, J. Li, Y. Guan, A. Imran, and M. Azeem, "Monitoring biochemical indicators using machine learning techniques for an effective large for gestational age prediction model with reduced computational overhead," in International Conference on Frontier Computing, pp. 130- 137, Springer, 2018.

[4]. F. Akhtar, J. Li, Y. Pei, A. Imran, A. Rajput, M. Azeem, and Q. Wang, "Diagnosis and prediction of large-for-gestational-age fetus using the stacked generalization method," Applied Sciences, vol. 9, no. 20, p. 4317, 2019.

[5]. V. Verma, V. Chowdary, M. K. Gupta, and A. K. Mondal, "Iot and robotics in healthcare," in Medical Big Data and Internet of Medical Things, pp. 245269, CRC Press, 2018.

[6]. T Karthick, Snigdha Pandey, Gayatri Dutt, Tarunjot Singh Kohli, "Prediction of water quality and smart water quality monitoring system in IoT environment", International Journal of Pure and Applied Mathematics, 2018, vol 118, pp. 3969-3977

[7]. Vijayakumar, K.P., Pradeep Mohan Kumar, K., Kottilingam, K. et al. An adaptive neuro-fuzzy logic based jamming detection system in WSN. Soft Comput 23, 2655-2667 (2019). https://doi.org/10.1007/s00500-018-3636-5

[8]. M. A. Khan and K. Salah, "Iot security: Review, blockchain solutions, and open challenges," Future Generation Computer Systems, vol. 82, pp. 395411, 2018. 
[9]. Jaree Thongkam, Guandong Xu, Yanchun Zhang and Fuchun Huang 'Breast Cancer Survivability competitive neural networks. Canadian Conference on Electrical and Computer Engineering (Vol. 1, pp.289-293).

[10]. Dilip Roy Chowdhury, Mridula Chatterjee\& R. K. Samanta, An Artificial Neural Network Model for Neonatal Disease Diagnosis, International Journal of Artificial Intelligence and Expert Systems (IJAE), Volume (2): Issue (3), 2012.

[11]. M. Anandan, M. Manikandan, T. Karthick, "Advanced Indoor and Outdoor Navigation System for Blind People Using Raspberry-Pi," Journal of Internet Technology, vol. 21, no. 1, pp. 183-195, Jan. 2020. 